\title{
Correction to: Comparison of sleeve gastrectomy and Roux-en-Y gastric bypass after failure of gastric banding: a two-center study with a propensity score-matched analysis
}

\author{
Antoine Vallois ${ }^{1} \cdot$ Lionel Rebibo $^{2,3,4} \cdot$ Yannick Le Roux ${ }^{1} \cdot$ Abdennaceur Dhahri $^{4,5}$ - Arnaud Alves ${ }^{1,6}$. \\ Jean-Marc Regimbeau ${ }^{4,5,7}$
}

Published online: 27 October 2020

(c) Springer Science+Business Media, LLC, part of Springer Nature 2020

\section{Correction to: Surgical Endoscopy https://doi.org/10.1007/s00464-020-07809-9}

This article was updated to correct the author listing, where first and last names were reversed.

Publisher's Note Springer Nature remains neutral with regard to jurisdictional claims in published maps and institutional affiliations.

The original article can be found online at https://doi.org/10.1007/ s00464-020-07809-9.

Jean-Marc Regimbeau

regimbeau.jean-marc@chu-amiens.fr

1 Department of Digestive Surgery, Caen University Hospital, Avenue de la Côte de Nacre, 14033 Caen, France

2 Department of Digestive, Esogastric and Bariatric Surgery, Bichat Claude Bernard University Hospital, 46 rue Henri Huchard, 75018 Paris, France

3 Université de Paris, Inserm UMR 1149, 75018 Paris, France

4 SSPC (Simplification of Surgical Patients Care) - Clinical Research Unit, University of Picardie Jules Verne, 80054 Amiens, France

5 Department of Digestive Surgery, Amiens University Medical Center, 1 Rond-Point du Professeur Christian Cabrol, 80054 Amiens, France

6 Anticipe, INSERM U1086, Pôle de recherche du CHU de Caen, Centre François Baclesse, Avenue du Général Harris, 14076 Caen Cedex 5, France

7 Service de Chirurgie Digestive, CHU Amiens-Picardie, Site Sud, 1 Rond-Point du Professeur Christian Cabrol, 80054 Amiens Cedex 1, France 